\title{
Anaesthesia for dentistry in children
}

\author{
Gardner BM \\ Department of Anaesthesia, Chris Hani Baragwanath Academic Hospital, University of the Witwatersrand \\ *Corresponding author, email:Brian.Gardner@wits.ac.za
}

Anaesthesia and dentistry have a long mutual history, and have developed in parallel over a similar time period. The first sedation for dental extraction took place on 11 December 1844 when the dentist Horace Wells had his wisdom tooth removed under nitrous oxide sedation. ${ }^{1,2}$ This was 2 years before William Morton performed the first ether general anaesthetic. ${ }^{2}$

Since then, advances in anaesthesia have been mirrored by progress in the type of dental techniques possible. The discovery of local anaesthetic agents in particular enabled the advent of modern dentistry. ${ }^{1}$

Today the majority of dental work is performed under local anaesthesia alone. Patient satisfaction is certainly greatest when local anaesthesia alone is used. An old study by Jeffrey Camm and colleagues showed very elegantly that the incidence of paediatric patient behavioural changes as well as parental stress were both similar and much greater when children received either sedation or general anaesthesia as opposed to local anaesthesia only. ${ }^{3}$ There are still numerous instances where additional sedation or general anaesthesia is required, however. Most notably these include all children under 10 years of age.

While the provision of general anaesthesia clearly falls within the realm of the anaesthetist, this distinction is not always so obvious with sedation, particularly in the minds of our dental colleagues. Dentists in the United States of America (USA) in particular have been extremely resistant to involve the anaesthetist in dental room sedation. Tragically, this has become topical because of the recent high number of adverse events including death in the USA. Typically these have been ASA 1 children.

Alarmingly the situation in South Africa is not that different. In 2015 Faizel Bham performed a study to audit dental chair sedation practice in Gauteng. ${ }^{4}$ Following random sampling, 213 dentists were interviewed. Ninety-four (44.1\%) of them routinely practised paediatric dental chair procedural sedation and analgesia (PSA). This group of 94 dentists was asked to complete an online survey, to which 52 (55.9\%) responded. The results were as follows:

- The overall PSA rate offered is $44.1 \%$.

- Most patients are $1-5$ years old. (76.6\% < 6 years).

- Minimal to moderate sedation endpoints are targeted.
- Midazolam is the most frequently used agent. This is occasionally supplemented with Nitrous Oxide (N2O).

- More than 3 agents are used in $28.1 \%$ of cases.

- A pre-sedation general medical assessment was only done in $83 \%$ of cases.

- Informed consent was only obtained in $75.6 \%$ of cases.

- No monitoring was used at all in $41.3 \%$ of cases.

- The dentist was the sole operator surgeon and sedationist in $41.3 \%$ of cases.

- No emergency drugs were available in $43.2 \%$ of cases.

- No emergency or resuscitation equipment was available in $19.76 \%$ of cases.

These terrifying results show extreme non-compliance with the South African Society of Anaesthesiologists (SASA) sedation guidelines. In the words of Dr De Wet of the Society of Sedation Practitioners of South Africa (SOSPOSA), "This is exactly how sedation should not be done".5

Where do problems arise? In 2000, Charles Cote analysed the contributing factors to adverse critical incidents in paediatric sedation. ${ }^{6}$ He used data from 3 sources:

1. The Food and Drug Administration (FDA) voluntary reports between 1969 and 1996.

2. The United States Pharmacopoeia (USP) drug events reports from a similar period.

3. Voluntary survey results obtained from a group of over 1300 USA practitioners.

Adverse critical events were divided according to where they occurred, i.e. hospital based vs non-hospital based settings. Under-reporting did likely influence their results. However, a total of 118 cases of adverse events involving sedation for any procedures in children were identified. In 95 of these cases the adverse event was purely due to the sedation. This is a low number but of these 95; 51 were deaths and 9 suffered permanent neurological damage, showing that when adverse events do occur they are catastrophic.

While the incidence of respiratory depression was equal in the hospital vs the non-hospital group, all other events were more common in the non-hospital setting: 


\begin{tabular}{lcc}
\hline & Non-hospital setting & Hospital setting \\
\hline Respiratory events & $\approx 80 \%$ & $\approx 80 \%$ \\
\hline $\begin{array}{l}\text { Cardiovascular events } \\
\text { (as 2nd complication) }\end{array}$ & $53.6 \%$ & $14 \%$ \\
$\begin{array}{l}\text { Cardiovascular events } \\
\text { (as 3rd complication) }\end{array}$ & $25 \%$ & $7 \%$ \\
\hline $\begin{array}{l}\text { Inadequate resuscitation } \\
\begin{array}{l}\text { Death or permanent } \\
\text { neurological injury }\end{array}\end{array}$ & $57.1 \%$ & $2.3 \%$ \\
\hline
\end{tabular}

Interestingly, the 35 patients who did not die or suffer from permanent neurological injury had all been monitored with pulse oximetry as opposed to the other cases, where no documented monitoring could be ascertained.

Of these 95 patients, 32 (33.7\%) had received sedation for dental procedures, pointing to the fact that across the full array of patients receiving sedation, dental procedures carry the highest risk.

Cote et al. reached the following conclusions:

1. The non-hospital setting carries a higher risk and is associated with poorer resuscitation and rescue chances.

2. Poor monitoring and poor responses to abnormal monitor readings are associated with poorer outcomes.

3. Pre-sedation assessments were often inadequately done or omitted.

4. Poorer outcomes correlate more frequently with single operator sedationists and where an independent observer was not present.

5. Medication errors in paediatric dosing were common.

6 . Inadequate recovery procedures were associated with poorer outcomes.

Charles Cote and colleagues also looked at the medications involved when these adverse events occurred. ${ }^{7}$ Looking at the 60 patients who suffered death or permanent neurological injury they found the following relationships:

\begin{tabular}{lc}
\hline & $\begin{array}{c}\text { Incidence of death or } \\
\text { permanent neurological injury }\end{array}$ \\
\hline Type of drug used. & No difference \\
Route of drug administration. & No difference. \\
Drug overdose. & $\Uparrow(28 / 60$ cases) \\
Drug combinations. & $\Uparrow$ (Especially if $\geq 3$ drugs used) \\
$\begin{array}{l}\mathrm{N}_{2} \mathrm{O} \text { added to other sedative } \\
\text { agents. }\end{array}$ & $\Uparrow$ \\
$\begin{array}{l}\text { Giving sedative to parent to } \\
\text { administer at home or en route } \\
\text { to the clinic. }\end{array}$ & $\Uparrow$ \\
$\begin{array}{l}\text { Non-medical sedationist or single } \\
\text { operator sedationist. }\end{array}$ & $\Uparrow$ \\
$\begin{array}{l}\text { Discharging patients home too } \\
\text { early before sedative(s) had worn } \\
\text { off. }\end{array}$ & $\Uparrow$ \\
\hline
\end{tabular}

Chicka et al. did a review of the closed malpractice insurance claims for dental related anaesthesia and PSA events, during the period 1993-2007 of two liability carriers. They discovered
17 claims. ${ }^{8}$ Of these, one patient had received a general anaesthetic and three received local anaesthesia alone. The remaining 13 had all received sedation. $53.6 \%$ of patients suffered death or permanent neurological damage. The average patient age was 3.6 years old. The dentist had been the sole operator sedationist and dentist in 6 of the 13 sedation cases. $71 \%$ of the sedation mishaps occurred in dental rooms. In 12 of the 13 sedation related incidents no monitoring had been used. Again, it was poor monitoring, single operator sedationist/ dentists and office-based sedation that stood out as risk factors.

Following all the media attention on deaths related to paediatric dental sedation or general anaesthesia, Lee et al. looked at media reports from 2 databases for the period 1980-2011. ${ }^{9}$ The databases used were the Lexis-Nexis Academic search engine and the Raven Maria Blanco foundation website. They specifically did not look at any outcome other than death (i.e. neurological damage or other adverse outcomes were excluded) and admit that under-reporting probably affected the accuracy of their numbers. A total of 44 deaths were found in these media reports. Forty-one of the patients were completely healthy prior to the event and all the deaths were believed to have been largely preventable. Three patients had pre-existing medical conditions which were not picked up during a pre-sedation assessment.

Lee found that:

- $21 / 44$ (47.7\%) of the patients were in the 2 to 5 -year age group.

- $21 / 44(47.7 \%)$ of the cases occurred in dental rooms.

- In 25/44 (56.8\%) of the cases the dentist had been a sole operator. In most of these cases moderate sedation had been targeted.

- Most patients initially suffered a respiratory arrest which was subsequently followed by a cardiac arrest.

- Sources of error were found to have been:

- An inadequate or no pre-sedation assessment was done.

- Medication errors.

- Inadequate or no monitoring was used during sedation.

- Inadequate resuscitation equipment was available and poor staff training in resuscitation skills.

Looking at dentistry itself, Nathan Reuter and his colleagues from the Department of Oral Health practice at the University of Kentucky, USA published a systematic review of death related to dental treatment in general in $2016^{10}$. They found dentistry itself to be very safe, with the mortality rate for pure, non-sedated dentistry to be less than 1 death per 10 million patients. However, as soon as PSA is added to the picture, this mortality rate jumps to around 1 death per 350000 patients. In fact, Reuter et al found $94 \%$ of all dentistry related deaths to be anaesthesia, sedation or medication error linked. They found the following associations:

- A strong association between death and when single service providers did the sedation as well as the dentistry.

- If a sedationist was present, then a strong link between the levels of training which the sedationist possessed and the type of outcome. 
- A strong association between death and deeper levels of sedation being used.

- Inadequate monitoring was a causal risk factor in most deaths.

- Poor emergency procedure implementation was common in the lethal cases.

- Children under 5 years were most at risk (most were normal, healthy children pre-procedure).

- Causes of respiratory related deaths were predominantly from airway obstruction due to:

- Foreign body aspiration.

- Angioedema.

- Hypersensitivity reactions.

- Spasm and asphyxia.

Looking at sedation in particular, Bellolio et al. did a systematic review and meta-analysis of 13883 PSA sedations done for children requiring minor procedures in the emergency department in 2016.11 These were all specifically non-dental cases. In contrast to studies which involve dental procedures, where death or permanent neurological damage are common, this meta-analysis revealed no deaths at all. In fact, respiratory complications were found to be very rare. These included 34 cases of laryngospasm, of which only one required intubation. This re-emphasizes the particular risk which PSA for dental procedures does carry.

The combination of PSA and dental procedures is particularly fraught. The greatest challenge is the shared airway. Minimal to moderate sedation is generally planned for, but there is always the risk of accidental over-sedation. The level of stimulation will fluctuate, making it difficult to maintain the sedation level constant without inadvertently slipping too far into the next level of sedation. Applying topical anaesthesia prior to inserting intravenous lines and dental local anaesthesia injections will help to avoid requiring a deeper sedation plane during these interventions. The use of $\mathrm{N}_{2} \mathrm{O}$ alone may be sufficient when there is no stimulation, but generally fails as soon as any painful or deep stimulation is given. The dentist must understand that having the patient in the correct plane of sedation means that the patient should still move. This will probably happen unexpectedly, either spontaneously but especially to painful stimuli. Aspiration of foreign bodies, blood or irrigation water is also a real danger and apart from Mendelsohn's syndrome, this may provoke laryngospasm and bronchospasm. The dentist must use the minimum amount of water to cool their drill and suction pedantically. Even with minimal to moderate PSA, most young children are still not able to tolerate sitting in the dental chair for very long. Often, only short, limited procedures are possible for these patients under PSA.

SASA has published updated guidelines for paediatric PSA in 2016. ${ }^{12}$ These guidelines cover the full ambit of PSA for all procedures in children, not only those presenting for dental procedures. SOSPOSA fully endorses these guidelines and is lobbying for the implementation of training and accreditation for sedationists in South Africa.
Sedation end-points are described as follows ${ }^{12}$ :

\section{Minimal sedation (Anxiolysis)}

Cognitive function is impaired but the patient responds normally to verbal commands. Ventilatory and cardiovascular function is unaffected.

\section{Moderate sedation}

The patient has a depressed level of consciousness but still responds purposefully to verbal commands or light touch. The patient maintains their own airway and breathing.

\section{Deep Sedation}

This forms part of the spectrum of general anaesthesia and may only be practised by practitioners with anaesthesia training.

Deep sedation is also known as "monitored anaesthesia care". The patient has a depressed level of consciousness but responds purposefully to repeated or painful stimuli (i.e. not merely a reflex withdrawal to pain).

The patient may not be able to maintain their own airway or breathing, which may require intervention by the sedationist.

\section{General anaesthesia}

The patient has a depressed level of consciousness from which they cannot be roused, even with painful stimuli.

The patient may not be able to maintain their own airway or breathing.

While minimal to moderate sedation is generally aimed for during PSA, it is important that the sedationist is always capable of rescuing a patient who inadvertently progresses to a deeper level of sedation.

In the SASA sedation guidelines a further distinction is made between simple or standard sedation and advanced sedation. SASA endorses single operator sedationist/dentists for the use of simple or standard sedation but requires a separate sedationist as soon as the use of advanced sedation is planned. Even with single-operator sedation, however, a separate observer must be present to monitor the patient. Simple sedation does also require that all safety equipment, monitoring and rescue protocols are in place. Non-fasted patients may be offered simple or standard sedation. Patients presenting for advanced sedation must all be fasted according to standard anaesthesia fasting guidelines. Fasting guidelines are currently a topic of debate in the literature. ${ }^{13}$ For now, however, the SASA guidelines remain 6 hours for solids and formula feeds, 4 hours for breast milk and 2 hours for clear fluids.

\section{Simple or standard sedation}

A single agent is used.

- This may be administered by an oral or transmucosal route.

- A single titrated intravenous dose of midazolam alone is permitted if Midazolam is used.

- Inhaled $\mathrm{N}_{2} \mathrm{O}$ in $50 \%$ oxygen only if $\mathrm{N}_{2} \mathrm{O}$ is used.

\section{Advanced sedation}

Comprises one or more of: 
- Any drug combination using more than one agent.

- An intravenous bolus injection or continued transfusions.

- Inhaled $\mathrm{N}_{2} \mathrm{O}$ in $<50 \%$ oxygen or any other inhaled agents used in oxygen instead of $\mathrm{N}_{2} \mathrm{O}$.

The SASA guidelines emphasize the importance of careful patient selection prior to performing $\mathrm{PSA}^{12}$ :

1. Only ASA I and II patients should be offered PSA. It is important to remember that an ASA II patient may deteriorate acutely and be ASA III on the day of the procedure, so a thorough presedation assessment is warranted.

2. All patients $<5$ years of age must be sedated by practitioners trained specifically in PSA for young children.

3. All PSA practitioners must have completed life support training.

4. Children suffering from an acute upper respiratory tract infection should be postponed until after this has resolved.

5. Children $<1$ year of age and all ASA III or higher patients should be referred to a specialist anaesthetist or highly experienced and trained paediatric sedationist. These patients should probably undergo their procedure in a hospital setting and not undergo an office-based procedure.

The importance of a thorough pre-sedation assessment cannot be overemphasised. Apart from identifying underlying medical conditions, a thorough focussed airway assessment must be done and the ability to communicate with the patient ascertained. It is important to ensure that the child will be able to cooperate and tolerate minimal to moderate sedation for the procedure.

Documentation collected must include:

- Informed consent;

- The pre-sedation assessment;

- The sedation chart outlining drugs administered and observed vital signs;

- Post-procedure observations;

- Home instructions.

Examples of all these documents are included in the SASA guidelines document. ${ }^{12}$ All the conditions required for safe day case procedures must also be adhered to.

Short-acting sedative agents are generally recommended and where applicable, antidotes must be available in the emergency drug armamentarium. Supplemental analgesia should not be forgotten. Most paediatric dentistry can be adequately managed with paracetamol and non-steroidal anti-inflammatory agents. Opioids are seldom required. The SASA guideline document includes a full description of suitable agents for PSA. ${ }^{12}$

Before office-based PSA is commenced, a clear plan for emergency management and transfer to a hospital setting must be in place. Emergency equipment and drugs required for rescuing a patient who proceeds to a deeper level of sedation, or who suffers an unanticipated complication, must be immediately available. A full list of these is available in the SASA guidelines document. ${ }^{12}$
The SASA guidelines for monitoring of minimal and moderate sedation include the following parameters:

1. Level of consciousness (University of Michigan Sedation Scale);

2. Breathing, ventilation and airway patency;

3. Heart rate and rhythm;

\section{Blood pressure;}

5. Oxygen saturation.

In patients with an underlying cardiovascular disease undergoing moderate sedation, the additional use of an electrocardiogram (ECG) is advised.

Capnography is not mandatory for moderate sedation but is recommended for deep sedation according to the SASA guidelines. There is always the risk of moderate sedation inadvertently progressing to deep sedation however, which is why recent lobbyists are advocating the use of capnography in all sedation cases. It is important to remember that the pulse oximeter is a lag monitor. Lightdale et al. of Children's Hospital in Boston, Massachusetts, did a randomised, controlled trial of the use of microstream capnography during moderate sedation in 2005 , challenging the guideline to monitor with pulse oximetry alone. ${ }^{14}$ They looked at the incidence of hypopnoea and apnoea diagnosed by observing chest movement and pulse oximetry vs capnography and their results were as follows for patients sedated with identical sedation protocols:

\begin{tabular}{lcc}
\hline & $\begin{array}{c}\text { Hypopnoea } \\
\text { detection rate }\end{array}$ & $\begin{array}{c}\text { Apnoea detection } \\
\text { rate }\end{array}$ \\
\hline $\begin{array}{l}\text { Chest wall } \\
\text { observation and } \\
\text { pulse oximetry only. }\end{array}$ & $3 \%$ detected & $0 \%$ detected \\
Capnography added. & $56 \%$ detected & $24 \%$ detected \\
\hline
\end{tabular}

Clearly we are simply not seeing the apnoea or hypopnoea episodes with our current observation methods if capnography is not used.

The addition of supplemental oxygen in particular may mask the desaturation required to register apnoea in a patient monitored by pulse oximetry alone.

While today paediatric dental PSA remains controversial, unlike in the 1970s and 1980s, the use of general anaesthesia for dental work in children is much more standardised, routine and safe. ${ }^{2}$ The main concerns are ${ }^{12}$ :

- Shared airway;

- Use of a throat pack;

- Emergence delirium;

- Outpatient setting considerations.

In conclusion, local anaesthesia used alone where possible remains the first choice for managing paediatric dental work. The use of general anaesthesia is safe, but costly and not always widely available. As an alternative, paediatric PSA for dental procedures is already being widely done, unfortunately, as can be seen from the Bham et al. study, often to poor safety standards in our country. Paediatric dental PSA is potentially 
extremely dangerous but the pitfalls and practice guidelines are quite clear. Education, accreditation and implementation of the SOSPOSA and SASA guidelines will hopefully go a long way to enhance safety for a practice which will likely remain popular, given the resource constraints we face.

\section{References:}

1. Cantlay K, Williamson S, Hawkings J. Anaesthesia for dentistry. Continuing Education in Anaesthesia, Crit Care Pain. 2005;5(3):71-5.

2. Mortazavi H, Baharvand M, Safi Y. Death Rate of Dental Anaesthesia. J Clin Diagn Res. 2017;11(6):7-9.

3. Camm JH, Mourino AP, Cobb EJ, et al. Behavioural changes of children undergoing dental treatment using sedation versus general anaesthesia. Pediatr Dent. 1987;9(2):111-7.

4. Bham F, Perrie $H$, Scribante J, et al. Paediatric dental chair sedation: An audit of current practice in Gauteng, South Africa. SAMJ. 2015;105(6):461-4.

5. De Wet. SASA refresher course lecture March 2017.

6. Cote CJ, Notterman DA, Karl HW, et al. Adverse Sedation Events in Pediatrics: A Critical Incident Analysis of Contributing Factors. Pediatrics. 2000;105(4):805-14.

7. Cote CJ, Karl HW, Notterman D, et al. Adverse Sedation Events in Pediatrics: Analysis of Medications Used for Sedation. Pediatrics. 2000;106(4):633-44.
8. Chicka MC, Dembo JB, Mathu-Muju KR, et al. Adverse events during pediatric dental anesthesia and sedation: a review of closed malpractice insurance claims. Pediatr Dent. 2012;34(3):231-8.

9. Lee $\mathrm{HH}$, Milgrom $\mathrm{P}$, Starks $\mathrm{H}$, et al. Trends in death associated with pediatric dental sedation and general anesthesia. Paediatr Anaesth. 2013;23(8):741-6.

10. Reuter NG, Westgate PM, Ingram $M$, et al. Death related to dental treatment: a systematic review. Oral Med. 2017;123(2):194-204.

11. Bellolio MF, Puls HA, Anderson JL, et al. Incidence of adverse events in paediatric procedural sedation in the emergency department: a systematic review and meta-analysis. BMJ Open. 2016 [accessed 12/04/2017];6:e011384:1-16. Available from: http://bmjopen.bmj.com/.

12. South African Society of Anaesthesiologists. Paediatric Sedation Guidelines for Procedural Sedation and Analgesia. SAJAA. 2016;22(1) Suppl 5:S1-33.

13. Green SM, Mason KP, Krauss BS. Pulmonary aspiration during procedural sedation: a comprehensive systematic review. BJA. 2017;118(3):344-54.

14. Lightdale JR, Goldmann DA, Feldman HA, et al. Microstream Capnography Improves Patient Monitoring During Moderate Sedation: A Randomized, Controlled Trial. Pediatrics. 2006;117(6):e1170-e1178.

15. Adewale L. Anaesthesia for paediatric dentistry. Continuing Education in Anaesthesia. Crit Care Pain. 2012;12(6):288-94. 